\title{
Improved approximation error bounds when modeling WLAN signals in flat fading channels
}

\author{
Christos Kalialakis ${ }^{\mathrm{a})}$ \\ EETT-Hellenic Telecommunications and Post Commission, \\ Thessaloniki Regional Office, Spectrum Department, \\ Thessaloniki State Airport, Thessaloniki, GR-551 03, Greece \\ a)C.Kalialakis@eett.gr
}

\begin{abstract}
The flat fading channel approximation error is investigated when employed for propagation channel modeling in wireless systems. The contributions of this paper are as follows. First, a bound for the approximation error is derived which is tighter in comparison with previously reported expressions. Second, practical error bounds are presented for WLAN systems in a typical indoor channel situation. The error bounds are derived as a function of the channel coherence bandwidth.
\end{abstract}

Keywords: flat fading, Wireless LAN, OFDM

Classification: Science and engineering for electronics

\section{References}

[1] [Online] http://standards.ieee.org

[2] R. Van Nee and R. Prasad, OFDM for Wireless Multimedia Communications, Artech House, Boston, 2000.

[3] P. Banelli, "Theoretical analysis and performance of OFDM signals in nonlinear fading channels," IEEE Trans. Wireless Commun., vol. 2, no. 2, pp. 284-293, March 2003.

[4] A. Ekbal, K. Song, and J. M. Cioffi, "QoS-Constrained Physical Layer Optimization for Correlated Flat Fading Wireless Channels," Proc. IEEE ICC, Paris, France, pp. 4211-4215, 2004.

[5] P. Hande, L. Tong, and A. Swami, "Flat Fading Approximation Error," IEEE Commun. Lett., vol. 4, no. 10, pp. 310-311, 2000.

[6] A. Saleh and R. Valenzuela, "A Statistical Model for Indoor Multipath Propagation," IEEE J. Select. Areas Commun., vol. 5, no. 2, pp. 128-137, 1987.

\section{Introduction}

Broadband wireless systems such as Wireless LAN (WLAN) have attracted a lot of interest. Several standards have appeared by IEEE under the 802.11 
family of standards like the 802.11b (also known as Wifi) and 802.11.a systems [1].

In general, flat fading is related to narrowband systems. However, many broadband wireless systems like WLAN and WiMax are using OFDM. In OFDM, through the use of subcarriers, the broadband signal spectrum is effectively divided in a number of subchannels each assumed to undergo flat fading [3]. What is termed as a narrowband signal depends on the channel response (coherence time) in relation with the signal data rate. This work is concerned to quantify the boundary between the narrowband and broadband regime in order to assess the suitability of the use of flat fading. Due to its simplicity, flat fading is utilised in performance evaluation given in closed form $[3,4]$.

In this paper, an improved average error bound estimate is derived which is tighter than the bound presented in [5]. The methodology is further applied to WLAN systems in an indoor channel model [6] and closed form average error bound expressions are presented as a function of channel coherence bandwidth.

\section{Error Formulation}

The aim of the analytical calculation is to derive time domain expressions. Then averages over channel statistics and time are evaluated. For completeness, the error formulation from [5] is revisited. The baseband representation of the received signal $r(t)$, in multipath fading over $\mathrm{L}$ paths is given by:

$$
r(t)=\sum_{n=1}^{L} \alpha_{n} s\left(t-\tau_{n}\right) e^{-j \psi_{n}}
$$

where $\psi_{\mathrm{n}}=2 \pi \mathrm{f}_{\mathrm{c}} \tau_{\mathrm{n}}(\mathrm{t})+\theta_{\mathrm{n}}, \theta_{\mathrm{n}}$ is a random phase distortion due to reflections and diffractions, $\tau_{\mathrm{n}}$ and $\alpha_{\mathrm{n}}$ are delay and attenuation parameters over the nth path respectively, $\mathrm{s}(\mathrm{t})$ is the baseband transmitted signal and $\mathrm{f}_{\mathrm{c}}$ is the carrier frequency.

In flat fading, the channel coherence bandwidth $\mathrm{B}_{\mathrm{c}}$, determined by the inverse of the channel delay spread $\tau_{\max }$, is large enough that the channel response becomes a multiplicative channel model. In such conditions, the multipath components are not resolvable meaning that all signal copies arrive at the same time. Therefore the differential path delays $\tau_{\mathrm{n}}$ are approximately equal to $\tau$. The flat fading condition can be expressed as

$$
s\left(t-\tau_{n}\right) \approx s(t)
$$

The flat fading condition Eq. (2) transforms Eq. (1) to the received signal under flat fading as

$$
\tilde{r}(t)=s(t) \sum_{n=1}^{L} \alpha_{n}(t) e^{-j \psi_{n}}
$$

The approximation error is defined as the difference between Eq. (1) and Eq. (3),

$$
e(t)=r(t)-\tilde{r}(t)
$$


The notion of average normalized error energy is used, defined in [5] as

$$
\overline{N E E} \equiv \frac{\int E\left[|e(t)|^{2}\right] d t}{\int E\left[|r(t)|^{2}\right] d t}
$$

where $\mathrm{E}[\mathrm{]}$ is the expectation operator.

The Normalized Error Energy is in general a random variable dependent on channel statistics and signal characteristics. The symbol NEE(t) will represent in this paper a channel averaged value. The averaged error expression results after integration of $\mathrm{NEE}(\mathrm{t})$ for the duration of at least one modulation symbol. In order to evaluate Eq. (5), expressions for the instantaneous squared error and square of the received signal are required.

Using Eq. (1) and Eq. (3) the error in Eq. (4) is expressed as,

$$
e(t)=\sum_{n=1}^{L} \alpha_{n} e^{-j \psi_{n}}\left[s(t)-s\left(t-\tau_{n}\right)\right]
$$

The error is a random variable because the channel parameters are random variables too. Taking into account that the signals are bandwidth limited, the baseband signal $\mathrm{s}\left(\mathrm{t}-\tau_{\mathrm{n}}\right)$ can be expanded using first order Taylor series. The instantaneous squared error is given by

$$
|e(t)|^{2}=|\dot{s}(t)|^{2} \sum_{k=1}^{L} \sum_{n=1}^{L} \alpha_{n} \alpha_{k} e^{-j \psi_{n}} e^{j \psi_{k}} \tau_{n} \tau_{k}
$$

where the dot denotes the time derivative.

The square of the received signal with no approximation $\mathrm{r}^{2}(\mathrm{t})$, treated in a similar manner, is expressed as

$$
|r(t)|^{2}=\sum_{n=1}^{L} \sum_{k=1}^{L} \alpha_{k}^{*} e^{j \psi_{k}} \alpha_{n} e^{-j \psi_{n}}\left[s^{2}-\tau_{n} \dot{s} s^{*}+\tau_{n} \tau_{k} \dot{s}^{2}-\tau_{k} s \dot{s}^{*}\right]
$$

\section{Channel Statistics and Averaging}

In general, any channel modeling can be considered as long as the distribution of time delays, attenuation and phase along each ray path are defined. Several models exist for WLAN. In the widely used indoor channel model presented in [6], the signal attenuations $\alpha_{\mathrm{n}}$ obey a Rayleigh distribution as follows

$$
E\left[a_{n}^{2} \mid \tau_{n}\right]=g_{n}^{2}=G e^{-\frac{\tau_{n}}{\gamma}}
$$

$\mathrm{g}_{\mathrm{n}}$ is the channel average amplitude attenuation and $\mathrm{G}$ the max amplitude, $\gamma$ a propagation parameter. The phase and the delay are distributed uniformly. Note that the delay spread $\tau_{\max }$ is connected with $\gamma$ because rays with attenuation larger than $\exp \left(-\tau_{\max } / \gamma\right)$ are not significant in magnitude. A useful choice of $\gamma$ that leads to ray attenuation of over $40 \mathrm{~dB}$ is $\gamma=0.1 \tau_{\max }$

Eq. (7) and Eq. (8) must be averaged over the channel statistics. The expectation operator $\mathrm{E}[]$ is first applied on Eq. (7), taking into account the channel statistics in Eq. (9), yielding

$$
E\left[|e(t)|^{2}\right]=|\dot{s}(t)|^{2} \frac{2 \gamma^{3} G L}{4 \pi^{2} \tau_{\max }}
$$


In a similar way, the expectation operator is applied to Eq. (8). $E[r(t)]^{2}$ is evaluated utilising standard exponential integrals leading to the following expression,

$$
E\left[|r(t)|^{2}\right]=\frac{L G \gamma}{4 \pi^{2} \tau_{\max }}\left[s^{2}(t)+2 \gamma^{2} \dot{s}^{2}(t)\right]-\frac{\gamma^{2} L G}{4 \pi^{2} \tau_{\max }}\left[\dot{s}(t) s^{*}(t)+s(t) \dot{s}^{*}(t)\right]
$$

\section{Error Bound}

Based on the methodology of the previous sections, the modulation dependent normalized error energy $\mathrm{NEE}(\mathrm{t})$ is expressed as the ratio of Eq. (10) and Eq. (11),

$$
N E E(t) \equiv \frac{E\left[|e(t)|^{2}\right]}{E\left[|r(t)|^{2}\right]}=\frac{2 \gamma^{2} \dot{s}^{2}}{s^{2}-\gamma \dot{s} s^{*}-\gamma s \dot{s}^{*}+2 \gamma^{2} \dot{s}^{2}}
$$

According to definition and the discussion in Section 2, for the indoor channel considered, Eq. (12) needs to be integrated over time. Analytical derivation can in principle be produced for any specific signal form. Alternatively, considering that the time integral is the energy of the signal and that according to Parseval's theorem an integral should yield the same value in time or frequency domain, the modulation masks can be interpreted as spectrum energy bound. Practical bounds can therefore be derived using spectrum masks. Using this argument, an upper bound was derived in [5] using a sinc baseband pulse which produces a rectangular spectrum mask. In this work, the integration of Eq. (12) is performed analytically in the frequency domain.

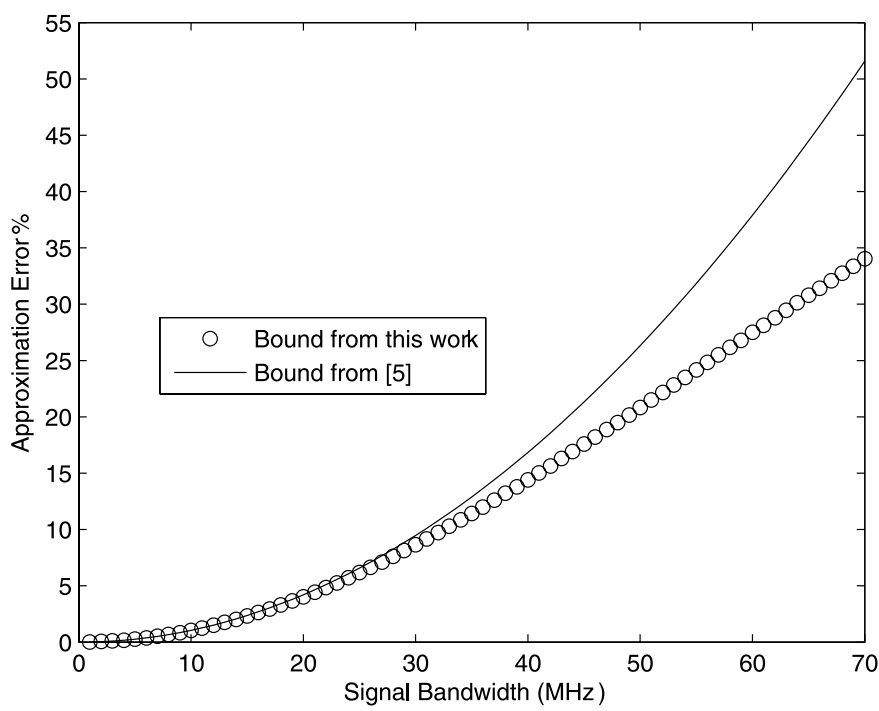

Fig. 1. Comparison of flat fading approximation error bounds as a function of signal bandwidth for a wireless channel with coherence bandwidth $50 \mathrm{MHz}$ (delay spread $20 \mathrm{~ns}$ ) 
Eq. (12) yields after integration an average bound,

$$
N E E_{\text {bound }}=\frac{\mathrm{B}^{2}}{3.8 B_{C}^{2}+\mathrm{B}^{2}}
$$

$\mathrm{B}$ represents the bandwidth of the wireless signal and $\mathrm{B}_{\mathrm{C}}$ is the coherence bandwidth of the channel.

Eq. (13) represents the maximum error introduced, when flat fading is used as a channel model, as a function of signal bandwidth and coherence bandwidth. This error bound is tighter when compared to the bound derived in [5]. The comparisons of the bounds are shown in Fig. 1 for the case of $\mathrm{t}=20 \mathrm{~ns}$ (equivalent to a channel of $\mathrm{B}_{\mathrm{C}}=50 \mathrm{MHz}$ ).

\section{Bounds for WLAN Systems}

Based on the argument in the previous section, the spectrum masks of WLAN modulation schemes are utilized. The concept is applied to 802.11a and 802.11b signal masks [1]. The bounds calculation requires piecewise integration of Eq. (13) over the frequency range of masks. The result as a function of coherence bandwidth, is for the 802.11 b system

$$
\overline{N E E}_{802.11 b}\left(B_{C}\right)=\frac{1}{0,026 B_{c}^{2}+1}
$$

and for the 802.11a system

$$
\overline{N E E}_{802.11 \alpha}\left(B_{c}\right)=\frac{1}{0.0234 B_{c}^{2}+1}
$$

where $\mathrm{B}=60 \mathrm{MHz}$ for 802.11. a and $\mathrm{B}=22 \mathrm{MHz}$ for $802.11 \mathrm{~b}$ were used.

The corresponding bound expressions for a sinc pulse having the same signal bandwidths as the WLAN signals are

$$
\begin{aligned}
& \overline{N E E}_{B=44 M H z}=\frac{1}{0,00196 B_{C}^{2}+1} \\
& \overline{N E E}_{B=60 M H z}=\frac{1}{0,0011 B_{C}^{2}+1}
\end{aligned}
$$

In Fig. 2 and Fig. 3 the error bounds for WLAN signal estimation are given when flat fading is used as a channel model. Eq. (14) and Eq. (15) provide tighter bounds than Eq. (16) and (17). It is to be noted that the 802.11.a bound is tighter compared to the 802.11 .b bound because its mask is concentrated in a smaller portion of the spectrum. Given the channel delay spread, Eq. (14), Eq. (15) provide the means to assess the suitability of flat fading channel modeling for WLAN systems. For example, consider an 802.11.a system signal in a channel with a coherence bandwidth of $28.5 \mathrm{MHz}$ (equivalent delay spread $25 \mathrm{~ns}$ ). A maximum error of $5 \%$ is introduced on the received signal estimation when the flat fading channel model is used. 


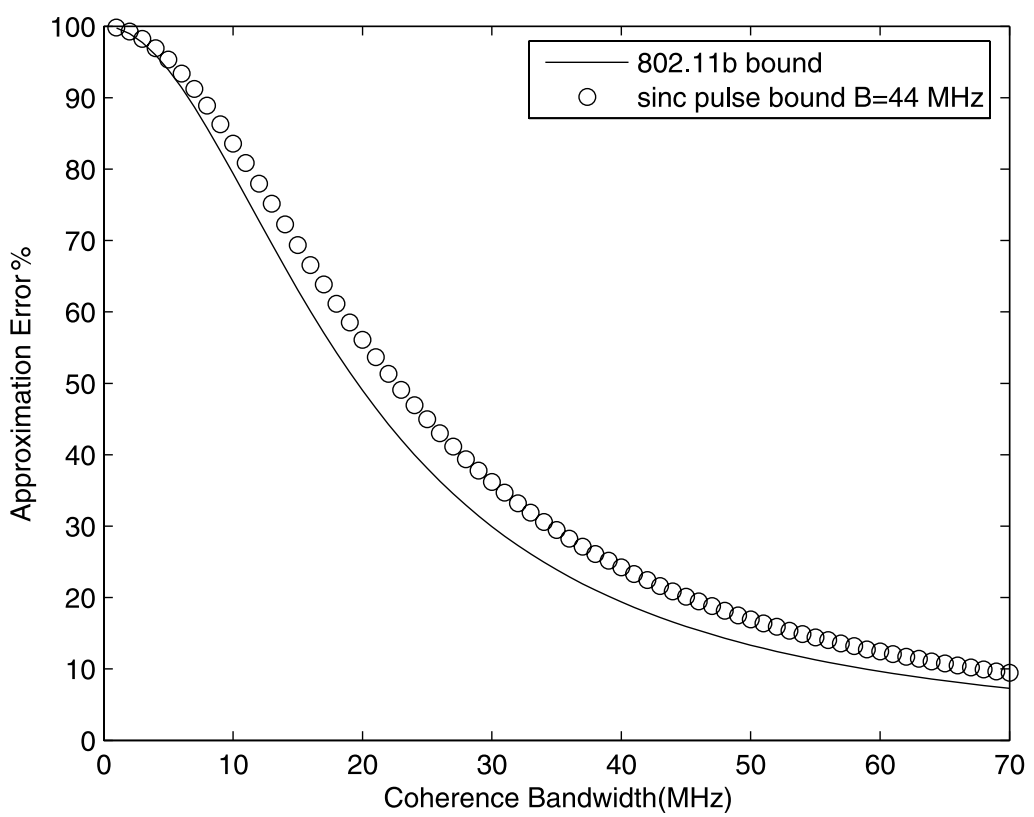

Fig. 2. Flat fading error bounds for WLAN 802.11.b signals compared to the previously used sinc pulse bound for $\mathrm{B}=44 \mathrm{MHz}$

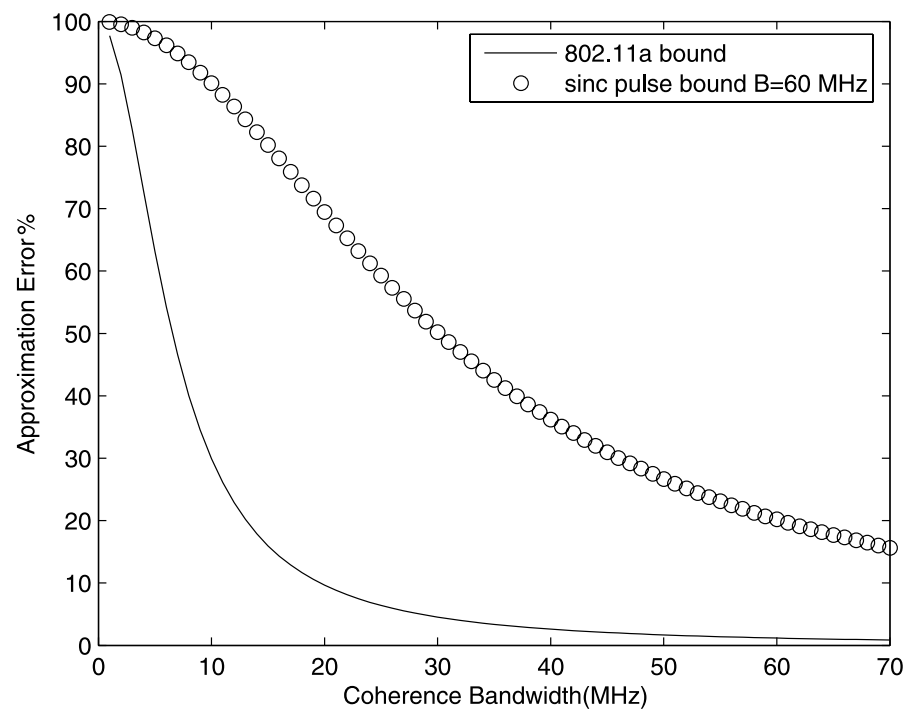

Fig. 3. Flat fading approximation error bounds for WLAN 802.11.a system compared to the previously used sinc pulse bound for $\mathrm{B}=60 \mathrm{MHz}$

\section{Conclusion}

A tighter bound has been introduced as a function of a wireless system signal bandwidth and the channel coherence bandwidth. Closed form expressions were presented for the error introduced in the received signal when the flat fading approximation is invoked for WLAN systems operating in indoor wireless channels. The methodology presented can be used to provide a solid guide to determine when a signal is narrowband or broadband in relation to 